\title{
COMPARAÇÃO DO DESEMPENHO FUNCIONAL DE CRIANÇAS PORTADORAS DE SÍNDROME DE DOWN E CRIANÇAS COM DESENVOLVIMENTO NORMAL AOS 2 E 5 ANOS DE IDADE
}

\author{
Marisa Cotta Mancini', Priscila Carvalho e Silva², \\ Sabrina Corrêa Gonçalves², Simone de Medeiros Martins²
}

\begin{abstract}
RESUMO - Objetivo: Comparar o desempenho funcional de crianças portadoras de síndrome de Down (SD) com crianças normais (DN), aos 2 e 5 anos de idade. Método: Quarenta crianças foram alocadas em quatro grupos $(n=10): 1$ ) crianças com SD de 2 anos; 2) crianças com SD de 5 anos; 3) crianças normais de 2 anos; 4) crianças normais de 5 anos. Todas foram avaliadas pelo teste funcional PEDI, que quantifica o desempenho infantil (habilidades e independência) em três áreas: auto-cuidado, mobilidade e função social. Testes de análises de variância fatorial foram utilizados para comparar médias dos grupos e testar fatores de interação idade x patologia. Contrastes pré-planejados foram usados para identificar as comparações bivariadas significativas. Resultados: Os fatores idadee patologia foram significativos nas três áreas de desempenho de habilidades e de independência. $\mathrm{O}$ fator de interação idade $x$ patologia mostrou-se significativo nas habilidades de auto-cuidado e mobilidade, e na independência em mobilidade e função social. Comparações bivariadas indicaram que aos dois anos, o desempenho de crianças normais é superior nas três áreas de habilidades funcionais e de independência. Entretanto, aos 5 anos, diferenças significativas entre os grupos foram observadas nas habilidades de autocuidado e de função social e na independência em auto-cuidado e em função social. Conclusão: Resultados informam áreas de desempenho onde o atraso apresentado por crianças com SD manifesta-se funcionalmente, aos dois e cinco anos de idade. Dados indicam que as diferenças observadas entre os dois grupos são influenciadas pela idade, não permanecendo constante ao longo do desenvolvimento.
\end{abstract}

PALAVRAS-CHAVE: síndrome de Down, desenvolvimento infantil, função.

\begin{abstract}
Comparison of functional performance among children with Down Syndrome and children with ageappropriate development at 2 and 5 years of age

ABSTRACT - Objective: To compare the functional performance of Down's syndrome (DS) children with normally developing children (ND), at 2 and 5 years of age. Method: Forty children were allocated into four groups $(n=10): 1)$ children with DS with 2 years of age; 2 ) children with DS with 5 years of age; 3 ) normal children with 2 years of age; 4) normal children with 5 years of age. Children were evaluated with the functional test PEDI, which quantifies children's performance (skills and independence) in three domains: self-care, mobility and social function. Two-way ANOVA was used to compare group means and to test interaction effects age $x$ pathology. Pre-planned contrast analyses were used to identify the significant bivariate comparisons. Results: Main factors (age and pathology) were significant in the three domains of skills and independence performances. The interaction factor age $x$ pathology was significant in self-care and mobility skills, as well as in children's independence in mobility and social function. Contrast analyses showed that at two years of age, normal children's performance is superior to DS's children in all three domains of functional skills and independence. However, at five years of age, significant group differences were only observed in the domains of self-care and social function skills, and independence. Conclusion: Results show the areas of performance where the delay presented by DS children was functionally manifested, at two and five years of age. Data indicate that the observed group differences were influenced by age, keeping themselves changeable across the development.
\end{abstract}

KEY WORDS: Down syndrome, child development, function.

Departamentos de Fisioterapia e de Terapia Ocupacional, Escola de Educação Física, Fisioterapia e Terapia Ocupacional, Universidade Federal de Minas Gerais, Belo Horizonte MG, Brasil (UFMG): ${ }^{1}$ Doutora em Ciências (Sc. D.), Professora Adjunta, Departamento de Terapia Ocupacional, UFMG; ${ }^{2}$ Fisioterapeuta, UFMG.

Recebido 7 Outubro 2002, recebido na forma final 18 Dezembro 2002. Aceito 13 Janeiro 2003. 
A Síndrome de Down (SD) é caracterizada como condição genética, que leva seu portador a apresentar uma série de características físicas e mentais específicas. Esta síndrome é considerada uma das mais frequentes anomalias numéricas dos cromossomos autossômicos e representa a mais antiga causa genética de retardo mental ${ }^{1-5}$. Dados epidemiológicos brasileiros revelam incidência de 1:600 nascidos vivos ${ }^{1}$. Avanços tecnológicos e científicos têm aumentado significativamente a sobrevida dessas crianças $^{4-7}$. Além disso, movimentos sócio-culturais têm buscado incluir estes indivíduos na sociedade, estimulando sua participação em diferentes contextos sociais, e promovendo o exercício da cidadania. Diversos estudos disponibilizam evidências sobre características do desempenho motor e cognitivo de crianças portadoras de $S^{1,3,4,8-12}$. No que se refere ao desenvolvimento de habilidades motoras, as evidências revelam que estas crianças apresentam atraso nas aquisições de marcos motores básicos, indicando que estes marcos emergem em tempo diferenciado (superior) ao de crianças com desenvolvimento normal ${ }^{1,3,4,8,9,12}$. A literatura também apresenta informações sobre o desempenho cognitivo de crianças portadoras de SD, indicando que estas crianças apresentam atraso ou retardo mental, que na verdade, é a manifestação de um sintoma desta condição genética ${ }^{3,7-9,11,12}$. Do ponto de vista cognitivo, observa-se um maior comprometimento destas crianças na área da linguagem ${ }^{1,3,4}$. As limitações motoras e cognitivas estão bem descritas na literatura, sendo que se observa uma predominância dos déficits motores no período referente à primeira infância e uma predominância dos déficits cognitivos na idade escolar ${ }^{1}$. Entretanto, a magnitude das diferenças do desempenho motor e cognitivo de crianças com SD em relação a crianças com desenvolvimento normal, pode não permanecer constante ao longo do desenvolvimento, caracterizando-se então, como uma hipótese a ser investigada.

As alterações apresentadas por crianças portadoras de SD podem se manifestar funcionalmente interferindo na capacidade destas crianças de desempenhar de forma independente diversas atividades e tarefas da rotina diária. Embora a literatura disponibilize evidências sobre as limitações conseqüentes desta condição genética em termos das funções de órgãos e sistemas que compõem a estrutura do corpo destas crianças (i.e. capacidades motoras e cognitivas), informações sobre o impacto destas limitações internas no desempenho de atividades diárias deste grupo são menos frequentes ${ }^{13}$. Entretanto, esse tipo de informação funcional é extremamente rele- vante para profissionais da área da saúde uma vez que as expectativas dos pais de crianças portadoras de SD estão mais relacionadas à informação funcional do que a informação sobre sintomatologia e componentes específicos de desempenho. A escassez de evidências sobre o desempenho funcional deste grupo clínico limita os profissionais que lidam com estas crianças a predizer desfechos e expectativas possíveis de serem alcançadas.

Este estudo transversal teve como objetivo comparar o desempenho funcional de crianças portadoras de SD com o de crianças que apresentam desenvolvimento normal, nas idades cronológicas de dois e cinco anos, em três áreas de desempenho: autocuidado, mobilidade e função social. Os resultados deste estudo poderão ser utilizados por profissionais da área de saúde para nortear os processos de avaliação e intervenção e informar aos familiares de crianças portadoras de SD sobre as prováveis consequências funcionais desta patologia, nas idades investigadas neste estudo.

\section{METODO}

\section{Participantes}

As quarenta crianças que participaram deste estudo foram escolhidas com base em critérios de inclusão e divididas em quatro grupos, conforme informações clínicas e grupo etário. Das 40 crianças, vinte eram portadoras de Síndrome de Down (SD) e as outras 20 crianças apresentavam desenvolvimento normal (DN), obedecendo aos seguintes critérios de inclusão:

Grupo SD: crianças diagnosticadas como portadoras de Síndrome de Down e com cariótipo determinado. Foram excluídas deste grupo aquelas crianças que apresentaram mosaicismo no cariótipo, problemas auditivos e/ou visuais. As crianças deste grupo foram selecionadas na Associação de Pais e Amigos dos Excepcionais (APAE), localizada no bairro Santa Tereza, em Belo Horizonte, e em instituições de ensino das redes pública e privada, da mesma cidade.

Grupo DN: crianças com desenvolvimento normal, sem problemas clínicos, diagnóstico de doença ou distúrbio do desenvolvimento e que não fizessem uso sistemático de medicação. As crianças deste grupo foram selecionadas na creche São Vicente de Paula, localizada no bairro Santa Tereza, em Belo Horizonte, e em instituições de ensino das redes pública e privada, da mesma cidade.

Cada um destes dois grupos de crianças (SD e DN) foi dividido ainda em outros dois sub-grupos etários, sendo um deles composto por crianças com idade cronológica entre 2 anos e 2 anos e 11 meses, e o outro constituído por crianças com idade entre 5 anos e 5 anos e 11 meses. Assim, as quarenta crianças que participaram deste estu- 
do foram alocadas em quatro subgrupos com dez crianças em cada. As duas faixas etárias (2 e 5 anos) foram selecionadas propositalmente, para diminuir o efeito diferenciado que períodos específicos impõem em áreas distintas de desempenho. Por exemplo, nos primeiros dois anos de vida, há um acelerado desenvolvimento observando-se grandes mudanças na área de mobilidade da criança. Após os cinco anos de idade, um grande número de crianças são iniciadas formalmente no processo de alfabetização e, consequentemente, diversas mudanças são observadas na área de função social (e.g., comunicação e compreensão). Além da estratificação por idade e patologia, a seleção das crianças para compor cada grupo foi feita de forma a buscar equivalência entre grupos também nas variáveis sexo e nível sócio-econômico da família.

As características sócio-econômicas das famílias foram definidas conforme critério proposto pela Associação Brasileira de Institutos de Pesquisa de Mercado - ABIPEME $(2001)^{14}$. A classificação sócio-econômica geral resultante deste critério varia de $\mathrm{A}$ (indicando classe sócio-econômica elevada) a $E$ (indicando classe sócio-econômica muito baixa), com categorias intermediárias ( $B, C$ e $D)$ indicando classes sócio-econômicas média e baixa.

\section{Instrumentação}

A avaliação das crianças foi feita utilizando-se o teste Pediatric Evaluation of Disability Inventory (PEDI) ${ }^{15}$ que é um instrumento padronizado norte-americano que documenta de forma quantitativa a capacidade funcional da criança (habilidades) e a independência para realizar atividades de auto-cuidado, mobilidade e função social. Esta avaliação foi desenvolvida para documentar o desempe- nho funcional de crianças na faixa etária entre seis meses e sete anos e meio de idade. Este teste foi traduzido para o português e adaptado conforme as características sócio-culturais brasileiras, com autorização dos autores ${ }^{16}$.

$O$ teste PEDI é administrado através de entrevista estruturada com o cuidador da criança com duração de 50 a 60 minutos. O teste é constituído de três partes, sendo que neste estudo, foram utilizadas as duas primeiras partes. A primeira parte do teste PEDI avalia as habilidades funcionais da criança para desempenhar atividades nas áreas de auto-cuidado (73 itens), mobilidade (59 itens) e função social (65 itens). Os escores recebidos em cada item (zero ou 1) são somados, produzindo três escores totais de habilidades funcionais.

A segunda parte do teste avalia a independência da criança para realizar tarefas funcionais nas mesmas três áreas: auto-cuidado (8 itens), mobilidade (7 itens) e função social (5 itens). Nessa parte, cada item é avaliado numa escala ordinal de 0 a 5, onde o escore 0 (zero) informa que a criança é totalmente dependente do cuidador na realização da tarefa funcional e o escore 5 indica que ela realiza a tarefa de forma independente. Escores intermediários informam sobre níveis diferenciados de ajuda fornecida pelo cuidador (máxima, moderada, mínima, supervisão). Nesta parte, os escores recebidos em cada item são somados, resultando em três escores totais de independência funcional ${ }^{15}$.

A terceira parte do teste informa sobre as modificações necessárias para o desempenho das tarefas funcionais nas mesmas três áreas descritas acima. Esta parte não foi utilizada neste estudo.

Tabela 1. Análise descritiva dos grupos de crianças com síndrome de Down (SD) e com desenvolvimento normal (DN), aos dois e cinco anos de idade, nas variáveis sexo, idade e nível sócio-econômico da família (NSE).

\begin{tabular}{|c|c|c|c|c|}
\hline \multirow[b]{3}{*}{ Variáveis } & \multicolumn{4}{|c|}{ Grupos } \\
\hline & \multicolumn{2}{|c|}{ Dois anos } & \multicolumn{2}{|c|}{ Cinco anos } \\
\hline & SD & DN & SD & DN \\
\hline Idade* & $2,07(3,26)$ & $2,067(3,59)$ & $5,043(3,85)$ & $5,048(3,68)$ \\
\hline \multicolumn{5}{|l|}{ Sexo** } \\
\hline Feminino & 5 & 5 & 4 & 4 \\
\hline Masculino & 5 & 5 & 6 & 6 \\
\hline \multicolumn{5}{|l|}{$\mathrm{NSE}^{* *}$} \\
\hline $\mathrm{A} 2$ & 2 & 2 & 0 & 0 \\
\hline B1 & 0 & 0 & 1 & 1 \\
\hline B2 & 1 & 1 & 0 & 1 \\
\hline $\mathrm{C}$ & 3 & 3 & 5 & 3 \\
\hline $\mathrm{D}$ & 4 & 3 & 4 & 5 \\
\hline $\mathrm{E}$ & 0 & 1 & 0 & 0 \\
\hline
\end{tabular}

* números indicam média (desvio padrão); ** números indicam frequência ( $\mathrm{N}$ ) de crianças em cada categoria. 
Tabela 2. Resultados das análises post-hoc utilizando contrastes pré-planejados para comparação dos grupos de crianças com desenvolvimento normal (DN) e com síndrome de Down (SD) nas habilidades funcionais de auto-cuidado, mobilidade e função social, aos dois e cinco anos de idade.

\begin{tabular}{|c|c|c|c|c|c|c|c|c|c|}
\hline \multirow{5}{*}{$\begin{array}{l}\text { Habilidades } \\
\text { funcionais }\end{array}$} & \multicolumn{9}{|c|}{ Grupos } \\
\hline & \multicolumn{4}{|c|}{ Dois anos } & & \multicolumn{4}{|c|}{ Cinco anos } \\
\hline & \multicolumn{2}{|c|}{ SD } & \multicolumn{2}{|c|}{$\mathrm{DN}$} & & \multicolumn{2}{|c|}{ SD } & \multicolumn{2}{|c|}{$\mathrm{DN}$} \\
\hline & Média & Desvio & Média & Desvio & & Média & Desvio & Média & Desvio \\
\hline & & Padrão & & Padrão & & & Padrão & & Padrão \\
\hline Auto-cuidado* & & & & & Auto-cuidado & & & & \\
\hline$F=52,37$ & 26,2 & 7,61 & 51,4 & 6,75 & $F=6,24$ & 57,7 & 10,09 & 66,4 & 6,1 \\
\hline$p=0,0001$ & & & & & $p=0,31$ & & & & \\
\hline Mobilidade* & & & & & Mobilidade & & & & \\
\hline$F=42,75$ & 29,6 & 10,1 & 51 & 3,92 & $F=3,82$ & 50,4 & 9,73 & 56,8 & 1,48 \\
\hline$p=0,0001$ & & & & & $p=0,05$ & & & & \\
\hline Função Social* & & & & & Função Social* & & & & \\
\hline$F=62,83$ & 21 & 7,16 & 45,6 & 4,9 & $F=28,26$ & 40,6 & 10,41 & 57,1 & 3 \\
\hline$p=0,0001$ & & & & & $p=0,0001$ & & & & \\
\hline
\end{tabular}

Tabela 3. Resultados das análises post-hoc utilizando contrastes pré-planejados para comparação dos grupos de crianças com desenvolvimento normal (DN) e com síndrome de Down (SD) na independência funcional em auto-cuidado, mobilidade e função social, aos dois e cinco anos de idade.

\begin{tabular}{|c|c|c|c|c|c|c|c|c|c|}
\hline \multirow{5}{*}{$\begin{array}{l}\text { Habilidades } \\
\text { funcionais }\end{array}$} & \multicolumn{9}{|c|}{ Grupos } \\
\hline & \multicolumn{4}{|c|}{ Dois anos } & & \multicolumn{4}{|c|}{ Cinco anos } \\
\hline & \multicolumn{2}{|c|}{ SD } & \multicolumn{2}{|c|}{ DN } & & \multicolumn{2}{|c|}{ SD } & \multicolumn{2}{|c|}{$\mathrm{DN}$} \\
\hline & Média & Desvio & Média & Desvio & & Média & Desvio & Média & Desvio \\
\hline & & Padrão & & Padrão & & & Padrão & & Padrão \\
\hline Auto-cuidado & & & & & Auto-cuidado & & & & \\
\hline$F=34,22$ & 6,4 & 3,6 & 23,8 & 8,18 & $F=11,08$ & 25,7 & 9,35 & 35,6 & 3,13 \\
\hline$p=0,0001$ & & & & & $p=0,03$ & & & & \\
\hline Mobilidade & & & & & Mobilidade & & & & \\
\hline$F=29,9$ & 18,3 & 7,82 & 31,4 & 2,63 & $F=2,013$ & 31,1 & 6,81 & 34,5 & 0,71 \\
\hline$p=0,0001$ & & & & & $p=0,05$ & & & & \\
\hline Função Social & & & & & Função Social & & & & \\
\hline$F=54,46$ & 5,7 & 4,57 & 20,5 & 4,67 & $F=18,34$ & 15,9 & 6,1 & 24,5 & 0,71 \\
\hline$p=0,0001$ & & & & & $p=0,0001$ & & & & \\
\hline
\end{tabular}

\section{Procedimento}

As avaliações foram realizadas por três examinadores que foram previamente treinados na aplicação do teste. Antes do início da coleta dos dados, a confiabilidade entre-examinadores foi avaliada, obtendo-se índices de consistência (I.C.C.) superiores a 0,90 em todas as seis escalas do teste. A avaliação de cada criança foi realizada num único momento através de entrevista com pais ou responsáveis que informaram sobre o desempenho da criança nas atividades e tarefas da rotina diária em casa ${ }^{15}$.

Antes das crianças serem incluídas neste estudo, seus pais ou responsáveis foram informados sobre os objetivos e procedimentos do mesmo e foram solicitados a as- sinarem um termo de consentimento para a participação das crianças. Este estudo foi aprovado pelo Comitê de Ética em Pesquisa da Universidade Federal de Minas Gerais (UFMG), em 28 de maio de 2002.

\section{Análise dos dados}

A descrição das crianças que compuseram a amostra deste estudo foi feita por meio de medidas de tendência central (média e desvio-padrão) e freqüência, nas variáveis idade, sexo e nível sócio-econômico da família.

Análises de variância fatorial (Two-way ANOVA) foram utilizadas para comparar as médias entre grupos, no que se refere às habilidades funcionais e a independência nas 


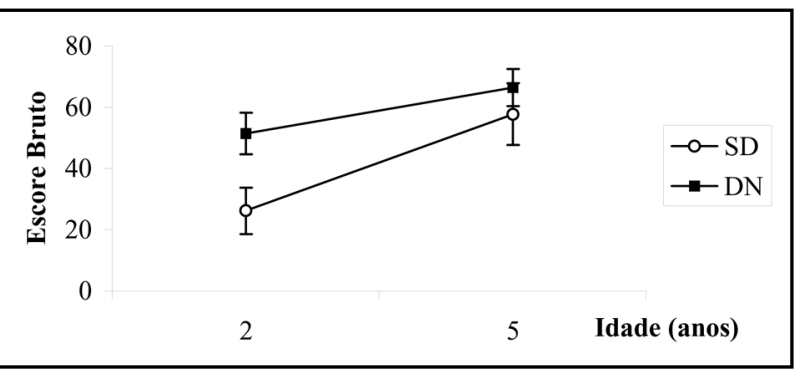

Fig 1. Gráfico ilustrativo do fator de interação idade x patologia nas habilidades funcionais de auto-cuidado $(p<0,05)$ em crianças com síndrome de Down (SD) e crianças com desenvolvimento normal (DN).

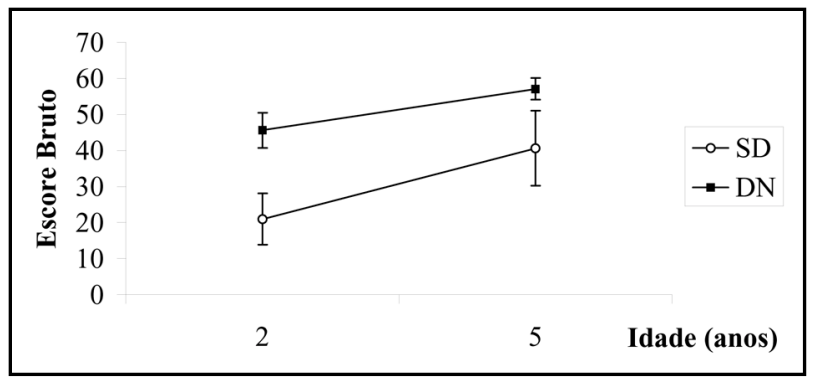

Fig 3. Gráfico ilustrativo do fator de interação idade x patologia nas habilidades funcionais de função social $(p>0,05)$ em crianças com síndrome de Down (SD) e crianças com desenvolvimento normal (DN).

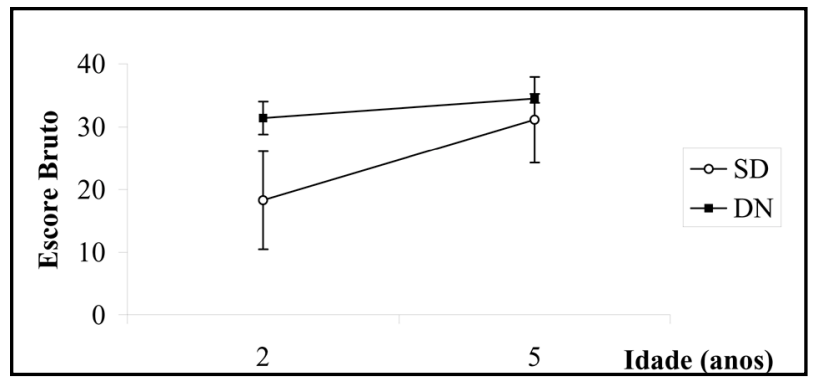

Fig 5. Gráfico ilustrativo do fator de interação idade x patologia na independência em mobilidade $(p<0,05)$ de crianças com síndrome de Down (SD) e crianças com desenvolvimento normal (DN).

três áreas de função (auto-cuidado, mobilidade e função social). Estas análises foram realizadas utilizando-se o pacote estatístico SUPER ANOVA. Este teste estatístico paramétrico foi selecionado para as análises inferenciais, pelo fato das variáveis dependentes deste estudo serem quantitativas (escala intervalar) e também pelo fato do modelo fatorial permitir a investigação do fator de interação (idade $x$ grupo $^{17}$. Quando o resultado do teste ANOVA revelou existência de diferença significativa, contrastes pré-planejados foram realizados para localizar onde a diferença foi estatisticamente significativa. Em todas as análises foi considerado o índice de significância $\alpha=0,05$.

\section{RESULTADOS}

A Tabela 1 apresenta informação descritiva das crianças que compuseram os quatro grupos, em relação às variáveis sexo, idade e nível sócio-econômico das famílias.

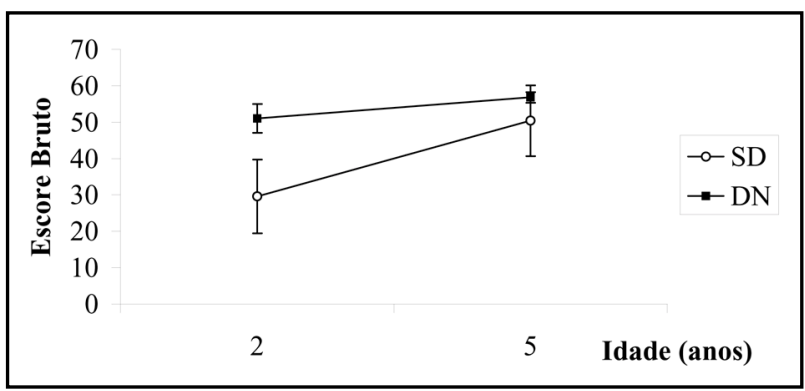

Fig 2. Gráfico ilustrativo do fator de interação idade $\mathrm{x}$ patologia nas habilidades funcionais de mobilidade $(p<0,05)$ em crianças com síndrome de Down (SD) e crianças com desenvolvimento normal (DN).

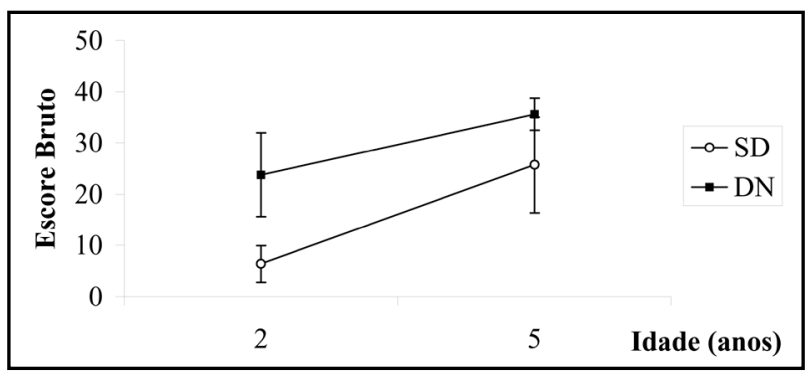

Fig 4. Gráfico ilustrativo do fator de interação idade x patologia na independência em auto-cuidado em crianças com síndrome de Down (SD) e crianças com desenvolvimento normal (DN).

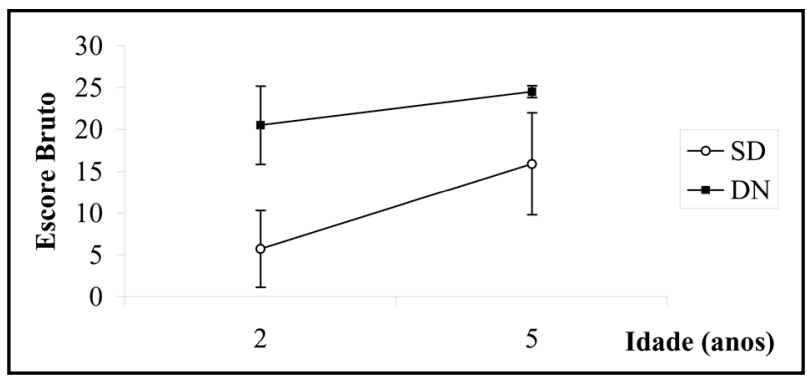

Fig 6. Gráfico ilustrativo do fator de interação idade x patologia na independência em função social $(p<0,05)$ em crianças com síndrome de Down (SD) e crianças com desenvolvimento normal (DN).

Análises de variância vatorial (Two-Way ANOVA) examinaram diferenças entre médias dos grupos nas três áreas de habilidades funcionais. Resultados destas análises revelaram diferenças significativas no fator patologia $\left(\mathrm{F}_{\text {auto-cuidado }}=47,39, \mathrm{p}=0,0001 ; \mathrm{F}_{\text {mobilidade }}\right.$ $\left.=36,07, p=0,0001 ; F_{\text {função social }}=87,69, p=0,0001\right)$. Além disso, o fator idade também apresentou diferença significativa nas habilidades de auto-cuidado $(\mathrm{F}=$ $89,17, p=0,0001)$, mobilidade $(F=33,02, p=0,0001)$ e de função social $(F=50,21, p=0,0001)$. Entretanto, o fator de interação idade $x$ patologia foi estatisticamente significativo nas habilidades de auto-cuidado $(F=11,23, p=0,0019)$ e de mobilidade $(F=10,50$, $p=0,0026)$, mas não apresentou significância estatística nas habilidades de função social $(F=3,406, p=$ 0,0732 ). As Figuras 1 a 3 ilustram os efeitos de interação nas três áreas de habilidades funcionais. 
Uma vez que diferenças significativas foram identificadas nos fatores de interação indicados aci$\mathrm{ma}$, contrastes pré-planejados foram utilizados para testar as diferenças entre grupos (DN e SD) em cada grupo etário (2 e 5 anos). Os resultados destas análises indicaram que aos dois anos de idade, diferenças entre crianças normais e com SD apresentaramse significativas nas três áreas de habilidades funcionais, entretanto, aos 5 anos de idade, diferenças significativas entre os dois grupos foi observada somente nas habilidades de auto-cuidado e de função social. A Tabela 2 apresenta os resultados das comparações post-hoc (contrastes) nas áreas de habilidades funcionais.

No que se refere à independência da criança ou quantidade de assistência fornecida pelo cuidador, observou-se diferenças significativas nas três áreas de função, tanto no fator patologia $\left(\mathrm{F}_{\text {auto-cuidado }}=\right.$ $42,12, p=0,0001 ; F_{\text {mobilidade }}=23,7, p=0,0001 ; F_{\text {função }}$ social $=68,07, p=0,0001)$ quanto no fator idade $\left(\mathrm{F}_{\text {auto- }}\right.$ cuidado $=54,66, p=0,0001 ; F_{\text {mobilidade }}=22,01, p=$ 0,$\left.0001 ; F_{\text {função social }}=25,07, p=0,0001\right)$. O fator de interação patologia $x$ idade apresentou significância estatística nas áreas de mobilidade $(F=8,19, p=$ $0,007)$ e de função social $(F=4,78, p=0,035)$. No entanto, não se observou significância estatística na independência em atividades de auto-cuidado $(F=$ $3,18, p=0,083)$. As interações referentes à assistência do cuidador nas três áreas de função estão ilustradas nas Figuras 4 a 6.

Os resultados das comparações post-hoc entre grupos (contrastes) referentes à independência, nas duas faixas etárias, estão representados na Tabela 3. Os dados revelaram uma diferença significativa entre os grupos de crianças normais e SD aos dois anos de idade, nas três áreas de independência funcional. No entanto, aos cinco anos, esta diferença apresentou-se significativa somente nas áreas de autocuidado e de função social.

\section{DISCUSSÃO}

Os resultados deste estudo demonstram que o desempenho funcional de crianças com SD é inferior ao de crianças normais. Entretanto, as interações entre patologia e grupo etário revelaram que este desempenho inferior não se mantém constante no contínuo do desenvolvimento. As crianças do grupo SD apresentaram um repertório de habilidades funcionais inferior e mostraram-se mais dependentes da ajuda fornecida pelo cuidador, quando comparadas com crianças com desenvolvimento normal, nas três áreas de função. Esses achados confirmam evidências existentes na literatura, no que se refere ao atraso no de- senvolvimento de crianças com SD $\mathrm{SD}^{1-4,8-12}$. No entanto, este estudo apresenta novas evidências que vêm acrescentar às informações existentes na literatura.

Foi observado que, apesar da diferença entre os desempenhos de crianças dos grupos SD e DN, essa diferença não permanece constante ao longo do desenvolvimento. Os resultados deste estudo mostram que a diferença entre os grupos apresenta-se mais evidente aos dois anos, e que, em algumas áreas de função, essa diferença é menos evidente aos cincos anos. Tal fato apresenta-se como uma maneira de se caracterizar o atraso apresentado por criança com SD, indicando que este atraso sofre influência da idade e, portanto, não deve ser entendido de forma linear.

A comparação do desempenho de crianças com SD e crianças normais demonstrou que na área de mobilidade, tanto nas habilidades funcionais quanto na ajuda fornecida pelo cuidador, há uma interação entre idade e patologia. Esta interação indica que, com o avançar da idade, o desempenho e a independência da criança com SD se aproximam do apresentado por crianças normais. Apesar dos grupos apresentarem desempenho em mobilidade significativamente diferente aos 2 anos, os resultados demonstraram que essa diferença não é significativa aos cinco anos de idade. Esta informação está de acordo com os resultados apresentados por Ramalho et al. ${ }^{4}$, que afirmam que o crescimento e o desenvolvimento de crianças com SD se aproximam daquele apresentado por crianças normais, no que se refere a habilidades motoras globais. Nossos resultados sugerem que, à medida que a criança com SD vai adquirindo habilidades na área de mobilidade, estas habilidades parecem ser incorporadas no repertório diário da mesma, ganhando também independência nesta área de função e recebendo menor assistência do seu cuidador.

$\mathrm{Na}$ área de auto-cuidado os resultados revelaram uma interação significativa entre patologia e idade nas habilidades funcionais, mas não na independência. Tal resultado mostra que a diferença de desempenho das crianças dos grupos DN e com SD não permanece constante nos dois grupos etários, observando-se uma distância maior aos dois anos e uma diferença menor aos cinco anos de idade. Por outro lado, no que se refere à independência das crianças nos dois grupos, observou-se que as crianças portadoras de SD são consistentemente menos independentes do que crianças normais tanto aos dois quanto aos cinco anos de idade. Pode-se argumentar que pais e cuidadores de crianças com SD tendem a limitar a participação destas crianças nas atividades de 
alimentação, banho, higiene pessoal, vestir e uso do banheiro (i.e. auto-cuidado), sugerindo uma superproteção dos mesmos. Outro fator indicado pelos pais no momento das entrevistas foi a falta de disponibilidade de tempo para permitir a participação destas crianças nas atividades de auto-cuidado. Este resultado fornece informação relevante para os profissionais que prestam atendimento a esta clientela, indicando que, no que se refere à independência para desempenhar atividades de auto-cuidado, os pais e cuidadores devem ser sensibilizados sobre a importância de permitir e estimular a participação de seus filhos nesta área funcional.

Outro resultado que merece ser discutido é a ausência de interação significativa entre patologia $x$ idade, nas habilidades de função social. Este resultado indica que o desempenho inferior apresentado por crianças com SD, comparados com crianças normais nas atividades que envolvem comunicação expressiva, compreensão, socialização e resolução de problemas, é observado tanto aos dois quanto aos cinco anos de idade. Tal fato ilustra a própria sintomatologia desta patologia que afeta o desempenho cognitivo destas crianças, e se manifesta funcionalmente nas atividades indicadas acima. Por outro lado, ainda na área de função social, observou-se que o fator de interação idade $x$ patologia foi estatisticamente significativo no que concerne a independência. Este resultado sugere que embora as habilidades de função social permaneçam inferiores nos dois grupos etários, crianças portadoras de SD apresentam-se mais independentes aos cinco anos que aos dois anos de idade, recebendo menor assistência do cuidador para compreender ordens, se comunicar e resolver problemas da rotina diária.

Uma limitação deste estudo refere-se à utilização de um desenho transversal para se avaliar diferenças de desempenho em dois perfis etários distintos. Apesar desta limitação, este estudo apontou questões importantes para a prática clínica no que se refere ao desenvolvimento de crianças com SD. Estes resultados ajudarão a desenhar um estudo longitudinal para avaliar e documentar as mudanças ao longo do desenvolvimento nestas crianças.

Os resultados deste estudo informam que as alterações apresentadas por crianças portadoras de SD se manifestam funcionalmente, influenciando na capacidade de desempenhar de forma independente diversas atividades e tarefas da rotina diária. Além disso, este estudo acrescenta novas informações refe- rentes à caracterização do atraso apresentado por estas crianças, indicando as áreas de desempenho e perfis etários onde este atraso é mais evidente. Tais informações podem ser úteis aos profissionais que trabalham com esta clientela, sugerindo áreas de ênfase nos processos de avaliação e intervenção em diferentes idades.

Agradecimentos - Agradecemos às instituições de ensino das redes pública e privada de Belo Horizonte, em especial à equipe da Clínica Intervir - APAE e à creche São Vicente de Paula, do bairro Santa Tereza, que colaboraram diretamente com o estudo facilitando o contato com os pais. Agradecemos também aos pais e responsáveis pelas crianças portadoras de síndrome de Down e crianças com desenvolvimento normal, que permitiram a participação de seus filhos neste estudo.

\section{REFERÊNCIAS}

1. Garcias G L, Roth M G M, Mesko GE, Boff T A. Aspectos do desenvolvimento neuropsicomotor na síndrome de Down. Rev Bras Neurol 1995; 31:245-248.

2. Schwartzman, J.S. Síndrome de Down. São Paulo: Editora Mackienze, 1999

3. Sarro KJ, Salina ME. Estudo de alguns fatores que influenciam no desenvolvimento das aquisições motoras de crianças portadoras de síndrome de Down em tratamento fisioterápico. Fisioter Mov 1999;8:93-106.

4. Ramalho C M J, Pedremônico M R, Perissinoto J. Síndrome de Down: avaliação do desempenho motor, coordenação e linguagem (entre dois e cinco anos). Temas sobre Desenvolvimento 2000;9:11-14.

5. Kasari C, Freeman S F N. Task-related social behavior in children with Down syndrome. Am J Ment Retard 2001;106 :253-264.

6. Ulrich BD, Ulrich DA, Collier D H, Cole E L. Developmental shifts in the ability of infants with Down syndrome to produce treadmill steps. Phys Ther 1995;75:14-23.

7. Leonard S, Bower C, Petterson B, Leonard H. Survival of infants born with Down's syndrome: 1980-96. Paediatr Perinat Epidemiol 2000; 14:163-171.

8. Connolly B H, Morgan S, Russel FF. Evaluation of children with Down syndrome who participated in an early intervention program. Phys Ther 1984;64:1515-1519.

9. Connolly B H, Michael B T. Performance of retarded children, with and without Down syndrome, on the Bruininks Oseretsky Test of Motor Proficiency. Phys Ther 1986;66:344-348.

10. ConnolyY B H, Morgan S B, Russel F F, Fulliton W L. A longitudinal study of children with Down syndrome who experienced early intervention programming. Phys Ther 1993;73:170-9;181

11. Mahoney G, Robinson C, Fewell R R. The effects of early motor intervention on children with Down syndrome or cerebral palsy: a fieldbased study. J Dev Behav Pediatr 2001;22:153-162.

12. Connolly B H, Morgan S B, Russel F F, Richardson B. Early intervention with Down syndrome children. Phys Ther 1980;60:1405-1408.

13. Organização Mundial de Saúde ICIDH-2: International classification of functioning and disability. Geneva: WHO; 2000.

14. Associação Brasileira dos Institutos de Pesquisa de Mercado (ABIPEME). Critério de classificação econômica Brasil www.targetmark.com.br/bf2001/br/criterio.htm

15. Haley S M, Coster W J, Ludlow L H, Haltiwanger J T, Andrellos PJ. Pediatric Evaluation of Disability Inventory (PEDI). Version 1.0. Boston, MA: New England Medical Center Hospitals, Inc.; 1992.

16. Haley S M, Coster WJ, Ludlow LH, Haltiwanger J T, Andrellos PJ. Inventário de avaliação pediátrica de disfunção - versão brasileira. Tradução e adaptação cultural: Mancini M C. Belo Horizonte: Laboratórios de Atividade e Desenvolvimento Infantil, Departamento de Terapia Ocupacional, Universidade Federal de Minas Gerais, 2000.

17. Portney LG, Watkins M P. Foundations of clinical research: applications to practice. 2.Ed. Upper Saddle River: Prentice Hall Health, 2000. 